\title{
Problems and Countermeasures in the Construction of Nosocomial Clinical Research Projects
}

\author{
Zijian $\mathrm{Wu}^{1, *}$, Lei Zhang ${ }^{1}$, Tiantian Cheng ${ }^{1}$, Shuting Ouyang ${ }^{1}$, Virginia Trigo ${ }^{2}$ \\ Zhihuan Yang $^{1}$ \\ ${ }^{1}$ Clinical Research Management Department, Cancer Centre of Guangzhou Medical University, No.78, Hengzhigang \\ Road, Yuexiu District, Guangzhou, Guangdong Province, China 510095 \\ ${ }^{2}$ Iscte Business School, BRU-IUL, University Institute of Lisbon, Avenida das Forças Armadas, 1649-026, Lisbon, \\ Portugal \\ *Corresponding author. Email: ngken@126.com
}

\begin{abstract}
In this paper, a case study was conducted in $\mathrm{C}$ hospital, which is a municipal university affiliated AAA hospital in China. We introduce its existed problems in the management of clinical research projects, the countermeasures and the construction results. Through analysis, it is found that the problems in the hospital mainly include: no dedicated department for the management; financial and incentive support are limited; lack of human resources, venues and information systems, etc.Starting in 2018,Chospital has adopted a series of countermeasures successively including: establishment of clinical research management department, establishment of phase I clinical trial ward and a clinical research fund; making full use of vertical and horizontal funds; offering more personnel and technical support. All the countermeasures formulated aim at helping the development of nosocomial clinical research projects. After more than 2 years of efforts, the hospital's scientific research achievements in quality and quantity are significantly increased than before.
\end{abstract}

Keywords: Clinical Research, AAA Hospital, Problems, Countermeasures, Complete Set of Support

\section{INTRODUCTION}

Science and technology innovation has always been the core of China 's overall construction and development plan. From Comrade Deng Xiaoping 's concept that "Science and Technology are the First Productive Forces" to General Secretary of the CPC Xi Jinping 's blueprint for the Chinese Dream, they highlight the role of technological innovation as a driver of rapid development of the country. Amid China's efforts to implement medical system reform and work to improve medical services, establishing research and innovation-oriented hospitals and using research and innovation to improve the overall hospitals' medical education and research have become the main development direction of medical institutions today ${ }^{[1]}$.

It is necessary for high-level large hospitals in China to build their strength in scientific research, and they have always been doing $\mathrm{so}^{[1-4]}$. With breakthroughs in basic life sciences, research on the mechanism of disease occurrence and development has gone further. The future disease diagnosis and treatment will gradually become more timely, accurate and intelligent ${ }^{[5]}$. Clinical research has become a key part in the chain of medical technology and innovation, providing an important foundation for the further transformation of results in basic life sciences and medical research into clinical applications, and the insurance of safety and efficacy of clinical diagnosis and treatment technologies and products ${ }^{[6]}$. This article takes the Hospital C, one of China's top-level cancer hospitals, as the research object, analyzes its existing problems, and figures out the solutions and their effects according to the clinical research project development and management.

\section{CLINICAL RESEARCH'S SIGNIFICANCE AND NECESSITY}

Clinical research takes human / patients as major subjects, diagnosis, treatment, prognosis, etiology and prevention as the major content, medical service institutions as the major base, and multi-disciplinary personnel as participators, organizers and actors ${ }^{[7] \cdot}$ Medical institutions actively carry out clinical research and strengthen its management and development, which has a positive impact on improving hospital quality and advancing its development.

\subsection{Strengthen the Service Philosophy of Medical Workers}

Clinical researchers must always put the safety of subjects first, and must patiently and dutifully inform them and obtain their consent before conducting trials. Therefore, by launching clinical research projects, hospital can further enhance the service philosophy of medical workers, and build a new relationship between doctors and patients with mutual respect, understanding and trust, so as to ensure that clinical researches run smoothly. 


\subsection{Adapt to New Requirements of Big Public Hospital Reform}

Under the current trend of big public hospital reform, public hospitals are seeing a negative impact on their revenues after the end of the system of covering hospital expenses with drug sales and controlling the proportion of medical consumables. Hospital actively carries out clinical research, especially good clinical practice (GCP project), medical personnel's diagnosis and treatment become more standardized and precise, and drug administration is more reasonable, which can effectively reduce the proportion of medicines and consumables. This is consistent with the goals of medical service transformation in the new era.

\subsection{More Standard Diagnosis and Treatment and More Scientific Health Care}

Clinical research adopts scientific methods and standards to study and evaluate the causes of diseases, determine and evaluate the effects and benefits of diagnosis, treatment, and prevention measures ${ }^{[5]}$. It can also further determine the side effects of certain drugs and estimate drug safety and its impact on patients. This is either good for clinicians' quality, ability of diagnosis and treatment, and accumulation of experience, or sustainable development in the hospital's clinical diagnosis and treatment.

\section{MANAGEMENT SITUATIONS AND PROBLEMS IN CLINICAL RESEARCH OF THE HOSPITAL C}

The Hospital $\mathrm{C}$ is a top-level hospital affiliated to a municipal university. In recent years, its medical education and research work has proceeded rapidly and delivered pleasant results. Since being certified as a state-level drug clinical trial institution in 2015, the hospital has undertaken plenty of GCP projects. By launching such trials, it has successfully declared many scientific research projects. This to some extent has made up for weakness in the hospital's scientific research, has increased its success rate of declaration in provincial-level key disciplines and clinical key specialties, and has raised the popularity of its existing key disciplines within the province, and also made it win a place in pharmaceutical R \& D and innovation. . However, by the evaluation standards of the national and provincial clinical key specialists and high-level hospitals, the Hospital $\mathrm{C}$ is relatively underperformed and has fewer high-quality and representative clinical research projects compared with other national regional medical centers. It also lacks research achievements with international influence. The problems in the clinical research and development of the Hospital $\mathrm{C}$ are as follows:

\subsection{Lack of Uniformed Management Platform}

Before 2019, three departments of scientific research, medical section and GCP all involved in the management of the establishment of clinical research projects. Therefore, the hospital did not establish a specialized department for such management, leaving different research projects disordered in management. And uniformed management was absent in the overall procure, from system, process, SOP operation to project establishment, supervision, evaluation and assessment.

\subsection{Clinicians Lack Professional Training}

Although senior clinical staff in the hospital are experienced and professionally skilled, most of them as clinical researchers have not received systematic and standardized clinical epidemiology training and thus have obvious shortcomings in plan design and implementation, data analysis and interpretation of statistical results. Besides, young and middle-aged talents with advanced degrees are limited to seniority and industry influence, so it is more difficult for them to undertake projects. As a result, the number and quality of clinical research projects in the hospital are disappointing.

\subsection{Lack of Research Funds}

GCP projects are premarketing clinical trial projects of drugs, sponsored by pharmaceutical companies. The funding for such projects is pleasant and can ensure the smooth development of the project. However, short of sufficient people, money, and materials, and with no specific funding application channels for such projects, an increasing number ofinvestigator initiated trials, (IIT project) can only refer to the funding management model of basic scientific research, yet the requirements of this model are not in line with the laws and characteristics of clinical research. The clear-cut problem is: management of many funds for clinical research that are largely used for subjects and researchers are strictly limited, and not allowed as a budget, resulting in insufficient funds and thus unpleasant progress of projects.

\subsection{Lack of Improved Incentive Mechanism}

Not having effective, sound funding and incentive mechanisms and performance evaluation approaches in clinical research projects, the Hospital $\mathrm{C}$ failed to protect the basic interests of clinical staff and give sufficient respect for them, and could not mobilize the enthusiasm of major researchers and high-educated backbone clinical staff at young and middle age in engaging with clinical research while involving in diagnosis and treatment work. 


\subsection{Insufficient Software and Hardware such as People, Places and Information System}

\subsubsection{No PhaseI Clinical Research Ward}

Without the support of phase I trials, the hospital cannot carry out preliminary clinical pharmacology and human safety evaluation trials, which heavily limits the development of clinical research.

\subsubsection{Insufficient Personnel and Professional Support}

Heavy clinical workloads do not allow necessary energy to work on research, so it requires enough relevant assistants such as scientific research secretary and clinical research coordinators (CRC). At the same time, many research projects have not employed statistical experts to support research plan design, data analysis and other work because of lacking funds, which to some extent affects the rationality and reliability of the research results.

\subsubsection{Lagging Behind in Digital Development}

For the management of research projects, the hospital has not done well in promoting digital development since it was certified as a drug clinical trial institution in 2015, and many management processes are still manually operated. With limited human resources, traditional management models and methods are nowhere near meeting the requirements of efficient and qualified clinical research project management. A complete information system is badly needed.

\section{RESPONSES}

\subsection{Centralized Management}

The level of clinical research is an important indicator of the hospital's comprehensive capacity. To solve the existing problems, the Hospital C started planning in 2018 and established in 2019 a clinical research management center directly under the leadership of the hospital leaders, and thus creates a unified working mechanism and based on it, works to advance clinical research.

\subsection{Comprehensive Management}

Since the establishment of the Clinical Research Management Center in 2019, the hospital has actively promoted the center's construction in all respects. After 2019, the center has hired 6 workers, established 4 functional groups of management, quality control, pharmacy and coordinator, creating a scientific and service-oriented clinical research management team. All this has provided conditions for standard clinical research and collaborative innovation projects on a large scale.

\subsection{Improve Institutional Management}

Considering the hospital conditions and related laws and regulations, the hospital has built an effective management system and a standard operation procedure (SOP), and improved institutional systems for the management of IIT projects that had no system framework previously. Such efforts can ensure the fine, institutionalized and scientific management in the whole process from project establishment, supervision, evaluation to assessment.

\subsection{Guarantee the Use of Funds}

As a saying goes: "an army marches on its stomach." Hospital clinical research construction involves certain risks and takes a long time before efforts reward. Without sufficient financial guarantee, the hospital is unable to ensure sound operation of projects, achieve expected construction goals and even transformation of research results. Therefore, while adding to the funds for supporting research projects and considering the development goals of scientific research driving development, the hospital rationally and legally allocates all kinds of funds, and creates a sufficient and sound financial guarantee system to meet the needs of clinical research.

\subsubsection{Increase Funds in Clinical Research Projects}

In the 14th Five-Year Plan in 2019, combining other hospitals' mature experience and successful examples, the hospital set up a special fund for clinical research to encourage great strides in the hospital's clinical research project development. The special fund invests not less than 5 million yuan, plans to sponsor 50 research projects, and expects to achieve 5 high-level research results within 5 years. In addition, in order to ensure targeted investment and project quality, the hospital has clarified several points to ensure that high-quality projects with good design, innovative ideas, and sufficient support are supported to achieve satisfactory results:

1) To ensure the output of high-quality research results, it is more appropriate to set up special funds for forward-looking clinical research projects and those engaged with diagnostic and therapeutic interventions. Retrospective observational analysis projects are not included in this fund;

2) Set certain requirements for the qualification, experience, background and the like of major researchers. For example, major researchers should be at a high 
professional level, have ability to organize and coordinate, and possess at least senior job titles.

3) Research teams must have a certain research basis, sound team building, and sufficient personnel and research support, and be consistent with the needs of platform construction and special projects.

4) As to the assessment of eligible projects, apart from scientific design, innovation, risk-benefit ratio, significance and benefits of the results, feasibility and operability should also be focused on.

5) Considering the scale and expected influence of projects, the hospital will rate the selected research projects in terms of funding. Projects with scientific and reasonable design, large sample size, impressiveinnovation, wide coverage, and welcome expected results and benefits can receive more supporting funds.

6) In order to boost the building of projects and improve the efficiency of the output and transformation of research results, the projects that complete ahead of schedule can apply to complete project acceptance early. If research teams pass it, a new round of fund assistance can be applied for

7) For the planned projects, the hospital opts for strict and dynamic process supervision and management, which involves the key points such as data quality, observance of ethical principles, diagnostic and treatment standards and legality in the development of projects. As such, quality control mechanism will be formed in clinical research management department and major researchers.

\subsubsection{Fully and Reasonably Relocate Varied Funds}

In 2019, the Hospital C declared a municipal high-level clinical key specialty construction project and succeeded. The Municipal Health Committee injected 1 million yuan into this project, and then the hospital added 1 million accordingly. While following principles of the relevant documents and related requirements of development and management and focusing on clinical research of the whole hospital, the hospital formulates incentive management mechanism corresponding to the supporting budget and funds. Besides, it propels sub-disciplines to actively participate in the development of clinical research. By doing so, the hospital expects to better achieve the overall goals of key specialty construction through the output and transformation of research results. At the same time, the hospital hopes that this round of development achievements can provide a foundation for the next round declaration. In addition to such projects, the hospital should also be active in declaring projects in various specialties, disciplines and comprehensive platforms established by higher-level departments (such as university clinical key specialties, municipal TCM key specialty, municipal key laboratories, etc.) This can not only help the hospital earn its reputation and gain an opportunity of further development, but also actively employs the support of various longtitudinal research funding to continue to move the hospital scientific research platform forward. On the premise of ensuring the protection of the intellectual property rights of its research results, the hospital also refers to the mature experience outside the hospital and in the industry [8, 9], and encourages enterprises and social capital to grant funding for research projects of it; by cooperating with qualified enterprises and institutions, the hospital has received research funding, and in return also provides technical support and assistance to enterprises and institutions with their own scientific research results.

\subsection{Support Services such as Personnel and Technology}

"If a craftsman wants to do good work, he must first sharpen his tools." It requires personnel, technology, places and other supports to advance research projects and deliver the aimed results. Therefore, beyond the organization and supervision of the development of projects, the clinical research project management department hopes more to play a great role in maintaining, including various assistance, guidance and support in personnel and technology, so as not only do a good job in leadership and planning, but also uphold the spirit of service to provide better security ${ }^{[10]}$.

Since 2019, the department has established stable cooperative relations with more than 10 qualified and professional SMO companies and scientific research services. When research projects in the hospital needs more research assistants and clinical research coordinators, technicians in different professional fields, or support in information technology, statistical services and others, the project actors can submit appeals and applications to the Clinical Research Management Center. In the connection network established by the center, it will provide assistances as much as possible according to the system, including recruiting professionals, searching for information technology assistance or service support for varied technologies and professional knowledge.

\subsection{Implement Information Management System}

In 2019, the hospital incorporated the survey results of other hospitals and introduced a practical information management platform for drug clinical trial project management through tendering to fully leverage the existing resources and improve the efficiency of various departments of the clinical research institution. The information system extensively underpins a series of research works in the hospital: assist various departments of the clinical research institution to spot problems in time, effectively support the cooperation of all professional research teams, swiftly and effectively manage clinical research projects, check the progress and relevant 
information of all clinical research projects in real time, and support the continuous improvement of the hospital's professional research and quality control.

\subsection{Establish PhaseI Clinical Research Ward}

At the end of 2019, the hospital was actively establishing a phase I clinical trial research ward, in accordance with the requirements of the Standards on National Regional Medical Center of Cancer (National Health Committee [2019] 697). At the beginning of 2020, the hospital hired lead researchers with academic influence in this field, introduced talents in translational medicine, and gradually sent out young and middle-aged high-educated backbone researchers who are committed in clinical research to study abroad, so as to build a high-level clinical research and management team.

\section{ACHIEVEMENTS}

According to statistics, during the 12th Five-Year Plan (2010-2015), C Institute launched 246 scientific research projects, 20 of which were subsidized by the National Natural Science Fund (NSFC), and received subsidies of 22.306 million yuan; published 85 SCI papers. However, just in 2018-2019, the hospital has made remarkable achievements, including 112 scientific research projects, 28 of which were subsidized by the NSFC, and received 15.775 million yuan as a whole; published 146 SCI papers. In addition, during these two years, the hospital also obtained 7 invention patents, 21 utility model patents, 1 design patent, and 8 software copyrights. Based on scientific research results, the hospital also actively strengthened the construction of specialties. During the past two years, it has passed several declarations and obtained 1 municipal high-level clinical key specialty construction project, 1 clinical key cultivation specialty project, and 2 university clinical specialties. All this has further promoted the combination of basic research and clinical applications, and the integrated development of specialties and disciplines.

\section{CONCLUSION}

Combining with the hospital's situations, establishing a scientific management mechanism, and providing comprehensive supports are the main cornerstone of the hospital's clinical research development. On the basis of ensuring the quality and safety of health care, only by establishing a high-quality clinical research incubation platform can medical institutions achieve high-quality and high-level clinical research outputs, ensure that the hospital is at the frontier of practice and technological innovation, and lay a foundation for greater social and economic benefits.

\section{REFERENCES}

[1] Chang, G., Wang, Y. (2015). Translational medicine leads the development of research hospitals. China Research Hospital, 2 (6), 42-45.

[2] Du, Y., Wang, C., Yang, Z., Huang, K., Xian, S. (2011). The significance of carrying out international multi-center drug clinical trials for medical institutions. Journal of Chinese Medicine Management, (03), 223-224.

[3] Hong, Q. (2019). Management and supervision of horizontal research funds. Accounting Learning, (17), 186-187.

[4] Li, N., Zhan, Q. (2014). Technological innovation and competitiveness of research hospitals. China Research Hospital, (01), 66-70.

[5] Liu, Y. (2010). On the "loose" and "tight" degree of funds management of horizontal scientific research projects. Mining Engineering Research, 31 (4), 140-141.

[6] Yi, X. (2011). Develop translational medicine to lead the development of research hospitals. PLA Hospital Management Journal, (12), 1107-1109.

[7] Zhao, Y. (2006). Some thoughts on the innovation of clinical research. Journal of Peking University (Medical Science), 38 (2), 121-123.

[8] Zhou, M., Zhu, J., Lin, K. (2001). Rely on scientific and technological progress to take the road of science and technology. Chinese Journal of Medical Research Management, (04), 52-54.

[9] Zhu, J., Zhou, M., Lin, K. (2002). Sun yat-sen university cancer prevention and treatment center of science and technology. Bulletin of Chinese Cancer, (04), 8-10.

[10] Zong, S. (2004). The development of clinical research level in China will be promoted by utilizing the advantages of the institute. China Medical News, 19 (9), 3. 